\title{
On the Fluctuation Induced Excess Conductivity in Stainless Steel Sheathed $\mathbf{M g B}_{2}$ Tapes
}

\author{
Suchitra Rajput ${ }^{1,2}$ and Sujeet Chaudhary ${ }^{1}$ \\ ${ }^{1}$ Thin Film Laboratory, Department of Physics, Indian Institute of Technology Delhi, New Delhi 110 016, India \\ ${ }^{2}$ IIMT college of Engineering, Maha Maya University, Greater Noida, Uttar Pradesh 201306, India \\ Correspondence should be addressed to Suchitra Rajput; rajput.suchitra@gmail.com
}

Received 18 November 2012; Revised 10 February 2013; Accepted 11 February 2013

Academic Editor: Ram Katiyar

Copyright ( 2013 S. Rajput and S. Chaudhary. This is an open access article distributed under the Creative Commons Attribution License, which permits unrestricted use, distribution, and reproduction in any medium, provided the original work is properly cited.

We report on the analyses of fluctuation induced excess conductivity in the $\rho$ - $T$ behavior in the in situ prepared $\mathrm{MgB}_{2}$ tapes. The scaling functions for critical fluctuations are employed to investigate the excess conductivity of these tapes around transition. Two scaling models for excess conductivity in the absence of magnetic field, namely, first, Aslamazov and Larkin model, second, Lawrence and Doniach model, have been employed for the study. Fitting the experimental $\rho-T$ data with these models indicates the three-dimensional nature of conduction of the carriers as opposed to the $2 \mathrm{D}$ character exhibited by the HTSCs. The estimated amplitude of coherence length from the fitted model is $\sim 21 \AA$.

\section{Introduction}

Since the discovery of superconductivity in $\mathrm{MgB}_{2}$ there has been enormous research owing to its speculated potential applications. Though there has been extensive research on both scientific and technical aspects, but yet little attention was paid to the fluctuation induced [1-5] enhanced conductivity in $\mathrm{MgB}_{2}$. Superconductor transition broadening, induced by these fluctuations in the superconducting order parameter, is observed in various kinds of superconductors $[6,7]$. Such broadening is normally due to superconducting fluctuation derived from a low dimensionality, short coherence length, and high $T_{C}$. High $T_{C}$, short coherence length, and low carrier densities impart an excess conductivity due to thermal fluctuation in the superconducting order parameter. There have been numerous studies on excess conductivity and order parameter fluctuations in $\mathrm{YBa}_{2} \mathrm{Cu}_{3} \mathrm{O}_{7-y}$ (Y-123) [8-17], $\mathrm{Bi}_{2} \mathrm{Sr}_{2} \mathrm{CaCu}_{2} \mathrm{O}_{8+y}$ (Bi-2122), $\mathrm{Bi}_{2} \mathrm{Sr}_{2} \mathrm{Ca}_{2} \mathrm{Cu}_{2} \mathrm{Cu}_{3} \mathrm{O}_{10+y}$ (Bi-2223) [18-22], $\mathrm{Tl}_{2} \mathrm{Ba}_{2} \mathrm{CaCu}_{2} \mathrm{O}_{8+y}$ (Tl-2212) [23, 24], $\mathrm{HgBa}_{2} \mathrm{Ca}_{2} \mathrm{Cu}_{3} \mathrm{O}_{8+y}(\mathrm{Hg}-1223)$, and $\mathrm{HgBa}_{2} \mathrm{CaCu}_{2} \mathrm{O}_{6+y}(\mathrm{Hg}-$ 1212) [25] systems, but only few reports exist on the fluctuation studies [26-28] in $\mathrm{MgB}_{2}$, and those are performed in the presence of magnetic field.
The superconducting transition in the absence of fluctuations is characterized by a mean non zero value of the order parameter below the transition temperature and zero above the transition temperature. There are fluctuations in the order parameter both above and below the transition temperature, that is, there is a probability of Cooper pair formation even above the transition temperature. These fluctuations contribute to the various physical properties like electrical conductivity, diamagnetism and specific heat both above and below $T_{C}$ [29]. The effect of thermodynamic fluctuations on most of these properties is in the small range around $T_{C}$ and the study of fluctuation conductivity may also provide information regarding the critical region close to $T_{C}$. We now first provide a brief description of the model describing the fluctuation effects in a superconductor based on its resistivity behaviour. We shall than use this framework/model to understand the transport behaviour observed for the $\mathrm{MgB}_{2}$ tape samples near the critical region around their $T_{C}$. We would present the results of the fluctuation induced conductivity in $\mathrm{MgB}_{2}$ tape via resistivity versus temperature measurement. The analyses of dimensionality of the conduction of the carriers and the estimation of the coherence length amplitude from fluctuation studies are also presented. 


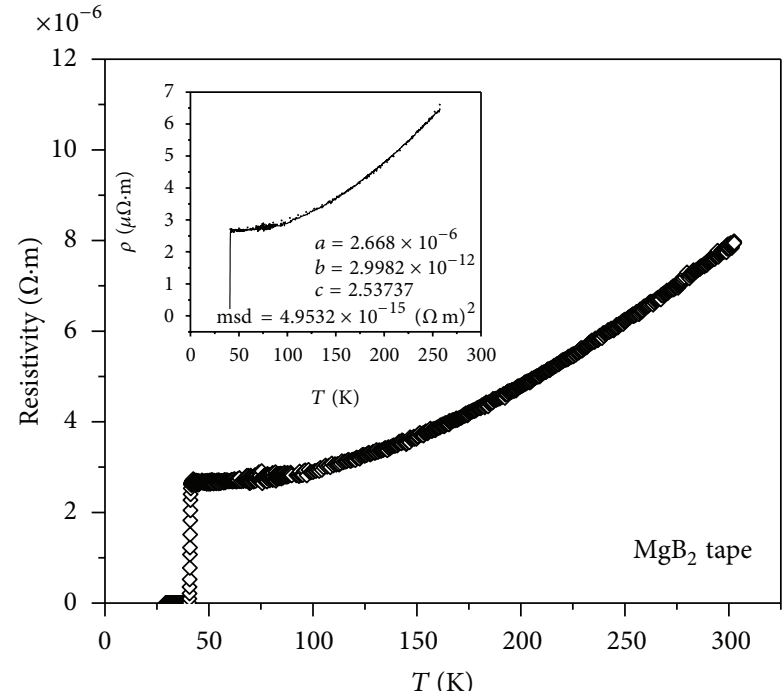

(a)

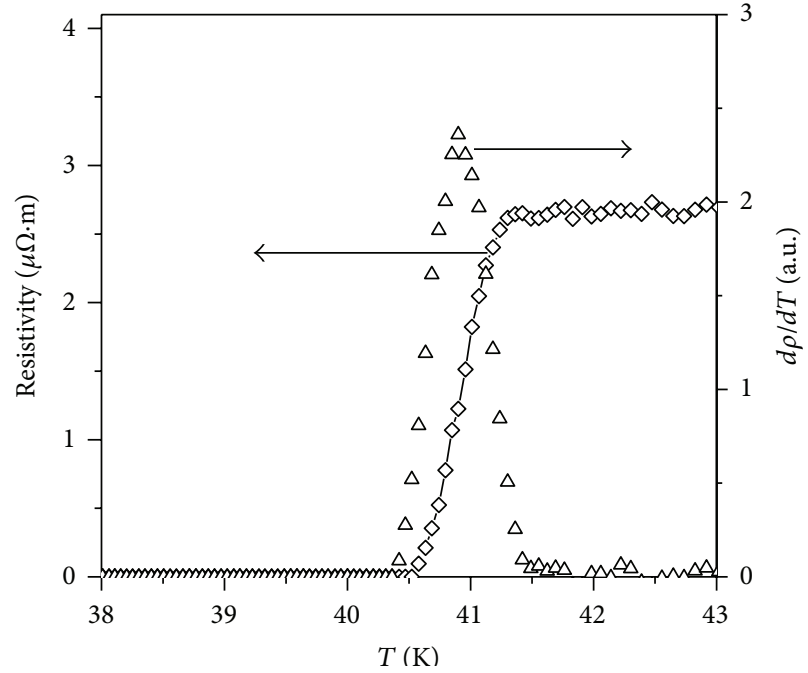

(b)

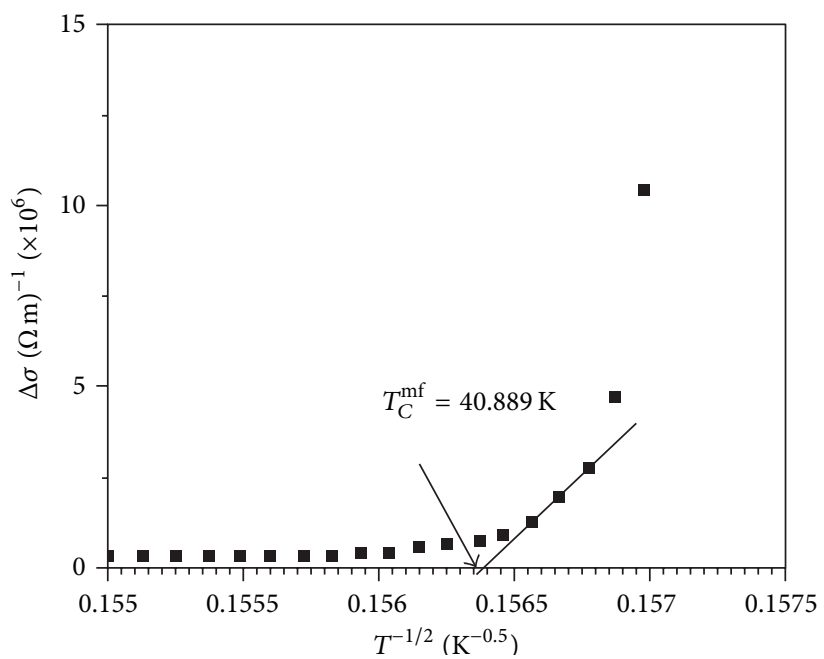

(c)

Figure 1: (a) Resistivity versus temperature curve for the in situ prepared stainless steel sheathed $\mathrm{MgB}_{2}$ tape sample. In the inset, experimentally observed data for resistivity with temperature has been shown by the dotted (..) curve, and the fitted data $\left(\rho_{n}(T)=a+b T^{c}\right)$ in the normal state has been shown by the solid (-) curve. (b) Variation of resistivity (diamond symbol with continuous line) and the differential of the resistivity $d \rho / d T$ (triangle) with the temperature for the in situ prepared stainless steel sheathed $\mathrm{MgB}_{2}$ tape sample. (c) $\Delta \sigma$ versus $T^{-1 / 2}$ plot for determining $T_{C}^{\mathrm{mf}}$, the linear extrapolated line hits the temperature where $\Delta \sigma$ starts tending to zero.

\section{Experimental Details}

For studying the fluctuation induced excess conductivity the in situ stainless steel sheathed tape sample synthesized at $800^{\circ} \mathrm{C}$, for $1 \mathrm{hr}$, described elsewhere [30], with the stoichiometric ( $\mathrm{Mg}: \mathrm{B}=1: 2)$ starting composition was prepared [30]. Silver pads for making four contacts on the sample were made via dc-sputtering method. After that the current and voltage contacts were made using copper wire and silver paste. The resistivity versus temperature data were recorded via the four-probe method while warming the sample with an accuracy of $\pm 1 \mathrm{mK}$ and at a rate of $0.1 \mathrm{~K} / \mathrm{min}$ around the transition, at the rate of $0.25 \mathrm{~K} / \mathrm{min}$ in the $50-100 \mathrm{~K}$ range, and at $0.5 \mathrm{~K} / \mathrm{min}$ in $100-300 \mathrm{~K}$ range. The temperature was recorded using a programmable temperature controller (Model-34 from Cryo-Con). A programmable current source (Model-224 from Keithley Inc.) was used to provide a constant current through the sample. Digital nanovoltmeter (Model181 from Keithley Inc.) having a high input impedance was employed to measure the potential difference developed when sample was slowly warmed/cooled between $15 \mathrm{~K}$ and room temperature.

\section{Result and Discussion}

3.1. Fluctuation Induced Excess Conductivity in the Absence of Magnetic Field. The initial studies on the fluctuation 


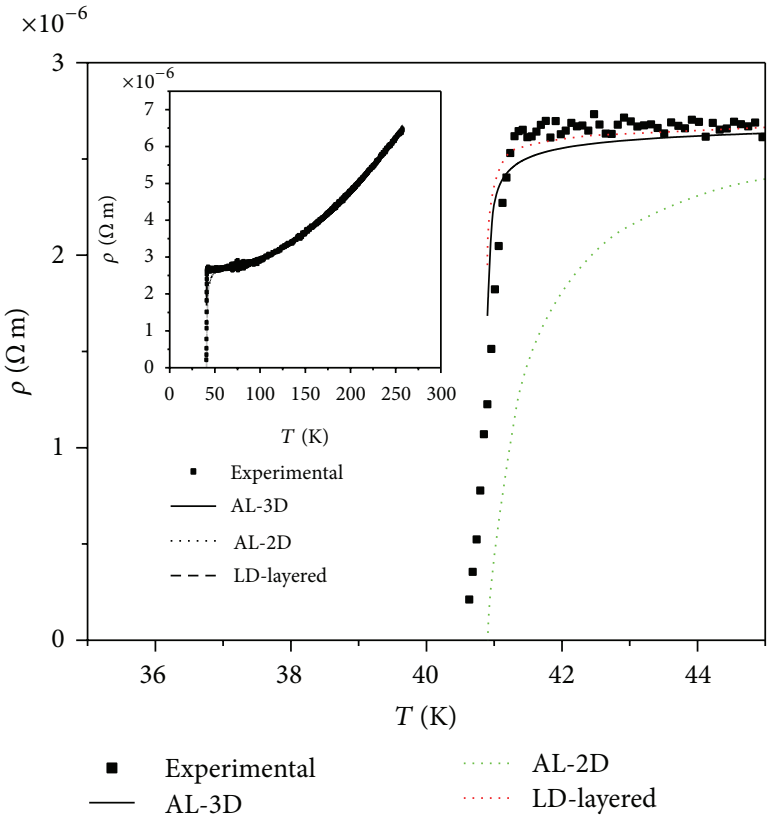

(a)

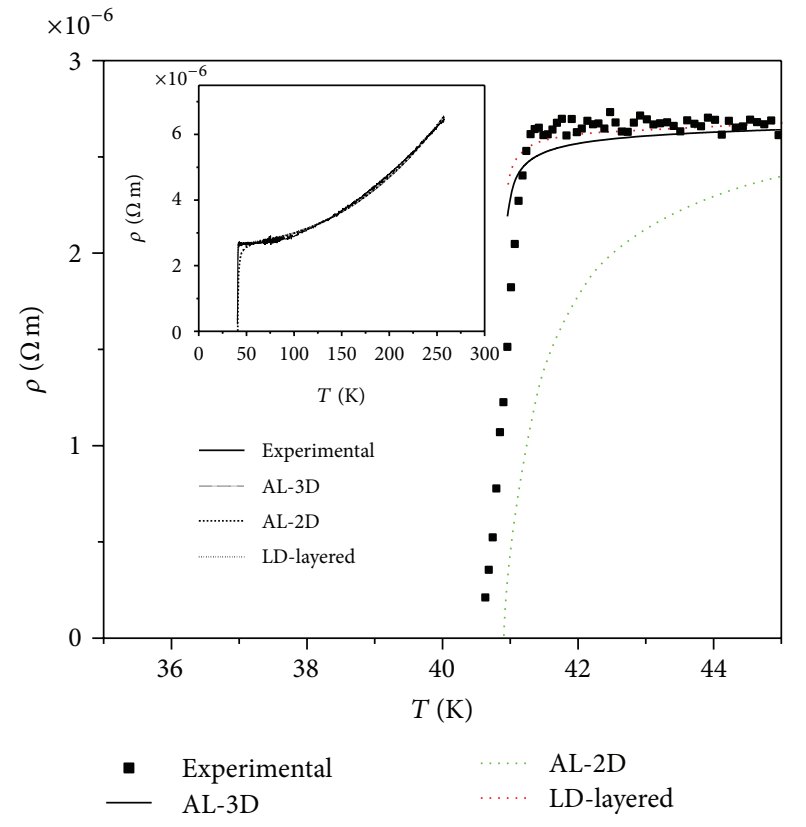

(b)

FIgURE 2: (a) Variation of resistivity versus temperature for $\mathrm{MgB}_{2}$ tape sample, experimental curve, and the fit to AL-2D, AL-3D, and LDlayered expressions with $T_{C}^{\mathrm{mf}}=40.889 \mathrm{~K}$ are shown near the superconducting transition. The inset shows the same curves over the wide range of temperature- $(15-250 \mathrm{~K})$. (b) Variation of resistivity versus temperature for $\mathrm{MgB}_{2}$ tape sample, experimental curve, and the fit to $\mathrm{AL}-2 \mathrm{D}$, AL-3D, and LD-layered expressions with $T_{C}^{\mathrm{mf}}=40.901 \mathrm{~K}$ are shown near the superconducting transition. The inset shows the same curves over the wide range of temperature- $(15-250 \mathrm{~K})$.

effects were performed by carrying out the measurement of excess conductivity above $T_{C}$, commonly known as the paraconductivity. Using the microscopic approach in the mean field region (MFR), Aslamazov and Larkin [31, 32], considering acceleration of the short lived superconducting charge carrier pairs which form in thermal equilibrium above $T_{C}$ in an electrical field, provided the expressions for 3- and 2-dimensional excess conductivity as follows:

$$
\begin{gathered}
\Delta \sigma_{3 \mathrm{D}}=\left(\frac{e^{2}}{32 \hbar \xi(0)}\right) \times \frac{1}{\varepsilon^{1 / 2}}, \\
\Delta \sigma_{2 \mathrm{D}}=\left(\frac{e^{2}}{16 \hbar d}\right) \times \frac{1}{\varepsilon},
\end{gathered}
$$

where, $\xi(0)$ is amplitude of isotropic coherence length, " $d$ " is the two-dimensional characteristic length of the sample, such that if $d \ll \xi \varepsilon^{1 / 2}$, then 2-dimentional excess conductivity dominates in the superconductor, and $\varepsilon$ is the reduced temperature defined as

$$
\varepsilon=\frac{T-T_{C}^{\mathrm{mf}}}{T_{C}^{\mathrm{mf}}}
$$

Here, $T_{C}^{\mathrm{mf}}$ is mean field temperature. In general, HTSCs are the highly anisotropic materials, and for such systems, Lawrence and Doniach [33] suggested a model with layered conduction in which conduction occurs mainly in twodimensional planes, and these planes are coupled through the well-known Josephson coupling. Within each layer, the superconductivity can be very well described by the GL theory. Lawrence and Doniach provided an expression for excess conductivity as

$$
\Delta \sigma_{L D}=\left(\frac{e^{2}}{16 \hbar s}\right) \times \varepsilon^{-1 / 2}(\varepsilon+4 L)^{-1 / 2} .
$$

Here $L=\left[\xi_{C}(0) / s\right]^{2}$ with $\xi_{C}(0)$ as the coherence length amplitude perpendicular to the planes, and $s$ is the distance between the planes. For $\varepsilon \gg 4 L$, that is, when $T \gg T_{C}^{\mathrm{mf}}$, this expression varies as $\varepsilon^{-1}$, thus showing the $2 \mathrm{D}$ behavior predicted by Aslamazov and Larkin (2). On the other hand, closer to $T_{C}^{\mathrm{mf}}$, that is, when $\varepsilon \ll 4 L$, this expression varies as $\varepsilon^{-1 / 2}$, thus presenting the $3 \mathrm{D}$ behavior (1). In other words, this formula interpolate between $2 \mathrm{D}$ formulation for $\xi(\varepsilon) \ll s$ (the interaction between layers is weak) and 3D (1) results for $\xi(\varepsilon) \gg s$, and a crossover 2D/3D is predicted at $\xi(\varepsilon) \sim s / 2$.

3.2. Resistivity-Temperature Behavior. Figure 1(a) presents the resistivity versus temperature $\rho(T, 0)$ curve for the in situ prepared stainless steel sheathed $\mathrm{MgB}_{2}$-tape sample $\mathrm{S} 800$. Figure 1(b) shows the enlarged view of the resistivity curve around the transition temperature and its derivative with the temperature. The single peak in the $d \rho / d T$ versus $T$ curve indicates that the sample is single phasic. The various parameters obtained from the curve in Figures 1(a) and 1(b) are the $\rho(300 \mathrm{~K})=7.92 \times 10^{-6} \Omega \mathrm{m}, \rho(42 \mathrm{~K})=2.68 \times 10^{-6} \Omega \mathrm{m}$, 
TABLE 1: Summary of the results obtained from fitting the resistivity data with AL and LD models with the normal state was fitted to $\rho_{n}(T)=$ $a+b T^{c}$.

\begin{tabular}{|c|c|c|c|c|c|}
\hline Criterion for $T_{\mathrm{C}}^{\mathrm{mf}}$ & $T_{\mathrm{C}}^{\mathrm{mf}}$ & Fitting model & $d$ or $s(\AA)$ & $\xi(0)(\AA)$ & $\operatorname{msd}\left(\sigma_{S}\right)(\Omega \mathrm{m})^{2}$ \\
\hline \multirow{3}{*}{ From linear extrapolation of $\Delta \sigma=0$} & \multirow{3}{*}{$40.889 \mathrm{~K}$} & AL-2D & 29.70 & - & $2.1 \times 10^{-12}$ \\
\hline & & AL-3D & - & 23.40 & $8.2 \times 10^{-14}$ \\
\hline & & LD-Layered & 40.87 & 35.3 & $1.3 \times 10^{-13}$ \\
\hline \multirow{3}{*}{ Peak in $d \rho / d T$ versus $T$ curve } & \multirow{3}{*}{$40.901 \mathrm{~K}$} & AL-2D & 30.76 & - & $2.1 \times 10^{-12}$ \\
\hline & & AL-3D & - & 21.05 & $6.1 \times 10^{-14}$ \\
\hline & & LD-Layered & 35.20 & 31.99 & $8.5 \times 10^{-14}$ \\
\hline
\end{tabular}

$\mathrm{RRR}=2.95, \Delta T(90 \%-10 \%$ criteria $)=0.50 \mathrm{~K}, T_{C}(90 \%$ drop criterion $)=40.663 \mathrm{~K}, T_{C}(10 \%$ drop criterion $)=41.189 \mathrm{~K}$, and $T_{C}$ (from peak in $\left.d \rho / d T\right)=40.901 \mathrm{~K}$. Here, RRR means residual resistivity ratio, namely, $\rho(300) / \rho(42)$ and $T_{C}(90 \%$ drop criterion), and $T_{C}$ (10\% drop criterion) corresponds to the temperature at which $\rho(42 \mathrm{~K})$ becomes $90 \%$ and $10 \%$, respectively.

The normal state behavior of the resistivity versus temperature curve of $\mathrm{MgB}_{2}$ tape $\mathrm{S} 800$ was fitted to $\rho_{n}(T)=a+b T^{c}$ above $1.5 T_{C}$ to $260 \mathrm{~K}$ (inset of Figure 1(a)) so as to obtain the $\Delta \sigma^{\mathrm{fl}}$ (i.e., fluctuation induced enhanced conductivity), which was found from the experimentally observed resistivity and the $\rho_{n}$ as

$$
\Delta \sigma^{\mathrm{fl}}(T)=\frac{1}{\rho(T)}-\frac{1}{\rho_{n}(T)} .
$$

From the fitting of the observed normal state $\rho_{n}(T)$ data, the constants $a, b$, and $c$ are found to be $2.6680 \times 10^{-6}, 2.9982 \times$ $10^{-12}$, and 2.5373 with msd (mean square deviation) between the experimental and the fitted data of $4.9327 \times 10^{-15}(\Omega \mathrm{m})^{2}$ (see inset of Figure 1(a)). The msd for any variable $f$ (varying as a function of $x$ ) is obtained by the following formula:

$$
\operatorname{msd}=\frac{\sum_{n}\left(f_{\text {experimental }}(x)-f_{\text {fitting }}\right)^{2}}{n} .
$$

The fitting of the observed excess conductivity $\Delta \sigma(T)$, obtained from the $\rho-T$ data with different models described previously, requires the estimation of the mean field temperature, $T_{C}^{\mathrm{mf}}$.

3.2.1. Mean Field Temperature. The choice of the mean field temperature is a well-known problem in the analysis of the fluctuation study. Various criteria for determining the $T_{C}^{\mathrm{mf}}$ have been considered in the literature $[9,34-36]$ as (i) midpoint of the resistive transition (ii) from the $90 \%$ drop in normal state resistivity near $T_{C}$ and (iii) the temperature at which $d \rho / d T$ versus $T$ curve exhibits a peak to use $T_{C}^{\mathrm{mf}}$ as a fitting parameter in minimizing the least square deviation between the observed and the predicted model. Notably, sometimes, $T_{C}^{\mathrm{mf}}$ is estimated from the linear extrapolation of $(\Delta \sigma)^{-2}=0$ in the $(\Delta \sigma)^{-2}$ versus $T$ plot (or $\Delta \sigma$ versus $T^{-1 / 2}$ plot). In the present work on $\mathrm{MgB}_{2}$, we have determined the $T_{C}^{\mathrm{mf}}$ from the $d \rho / d T$ versus $T$ curve and also from the $\Delta \sigma(T)$ data as suggested by $\mathrm{Oh}$ et al. [9], that is, from the $\Delta \sigma$ versus
$T^{-1 / 2}$ plot (see Figure 1(c)). The linear extrapolation of the plot to $\Delta \sigma=0$ has been considered to infer the corresponding temperature $T_{C}^{\mathrm{mf}}$.

In Table 1, we presents the various parameters obtained by fitting the measured resistivity data to different AL and LD models using (1)-(6). Figures 2(a) and 2(b) show the fitting of the experimental obtained resistivity versus temperature data with various models outlined previously using two values of mean field temperature $T_{C}^{\mathrm{mf}}$, respectively.

It is evident from Table 1 that the resistivity fit using AL model yields higher value of msd (namely, msd $=2.1$ $\times 10^{-12}(\Omega \mathrm{m})^{2}$ using $T_{C}^{\mathrm{mf}}=40.889$ and $\left.40.901 \mathrm{~K}\right)$ for $2 \mathrm{D}$ formulation of excess conductivity compared to the AL$3 \mathrm{D}$ model (namely, msd $=8.2 \times 10^{-14}(\Omega \mathrm{m})^{2}$ and $6.1 \times$ $10^{-14}(\Omega \mathrm{m})^{2}$ using $T_{\mathrm{C}}^{\mathrm{mf}}=40.880$ and $40.901 \mathrm{~K}$, resp.) for both the values of $T_{C}^{\mathrm{mf}}$ employed for fitting purpose. On the other hand, fit to LD-layered model yielded the msd value (namely, $\mathrm{msd}=1.3 \times 10^{-13}(\Omega \mathrm{m})^{2}$ and $8.5 \times 10^{-14}(\Omega \mathrm{m})^{2}$ using $T_{C}^{\mathrm{mf}}$ $=40.880$ and $40.901 \mathrm{~K}$, resp.) higher than that achieved in the case of AL-3D fit but lower than that for AL-2D fit. $\varepsilon$ values [37] corresponding to both the $T_{C}^{\mathrm{mf}}$ values have been found using specific heat jump $=64 \mathrm{~mJ} /(\mathrm{mol} \mathrm{K})$ [38] and $\xi_{C}(0)$ (from Table 1) and Coherence length anisotropy, $\gamma_{\xi} \sim 6$ [39]. It is found that $\varepsilon=1.94 \times 10^{-2}$ and $\varepsilon=$ $2.6 \times 10^{-2}$ corresponding to $T_{C}^{\mathrm{mf}}=40.889 \mathrm{~K}$ and $40.901 \mathrm{~K}$, respectively. Further, the corresponding $\xi(\varepsilon)$ comes out to be $\xi(\varepsilon) \gg s$ (namely, for $T_{C}^{\mathrm{mf}}=40.889 \mathrm{~K}, \xi(\varepsilon) \sim 253.9 \AA$ ), which clearly indicates the $3 \mathrm{D}$ fluctuation in the $\mathrm{MgB}_{2}$ tape sample. All these results show that the different observables around the $\mathrm{MgB}_{2}$ superconducting transition may be explained, in most of the experimentally accessible range, by considering Gaussian fluctuation in the mean-field Ginzburg-Landau like approaches. Thus, in the absence of magnetic field, the full critical region must then be expected only for temperatures close to mean field temperature (for $\left|T-T_{C}^{\mathrm{mf}}\right| \leq 1 \mathrm{~K}$ ). Here, it is worth mentioning that the msd values presented in Table 1 for various fitting are evaluated for the data in the previous range.

Thus, it may be concluded that fluctuation induced enhanced conductivity in $\mathrm{MgB}_{2}$ tapes fit to the 3D relation for enhancement of the conductivity near the transition regime. The fluctuation study in the zero field yields the amplitude of coherence length $\xi(0) \sim 21 \AA$ which agrees very closely to the value $(\sim 26 \AA)$ reported by Kim et al. [34]. These authors carried out the fluctuation study in the specific heat behavior 
in the bulk $\mathrm{Mg}^{11} \mathrm{~B}_{2}$ sample in absence of field, neglecting the inhomogeneity of the sample.

\section{Conclusions}

We have experimentally observed the fluctuation induced enhancement of conductivity in $\mathrm{MgB}_{2}$ in the absence of magnetic field. Study of fluctuation induced excess conductivity in the $\mathrm{MgB}_{2}$ sample via fitting of resistivity versus temperature data with Aslamazov and Larkin model and Lawrence and Doniach model indicates the three-dimensional nature of conduction of the carriers as opposed to the $2 \mathrm{D}$ character exhibited by the HTSCs. The estimated amplitude of coherence length from the fitted model is $\sim 21 \AA$.

\section{Conflict of Interests}

It should be noted that the authors (S. Rajput and S. Chaudhary) do not have any conflict of interests regarding the content of the paper.

\section{References}

[1] T. Park, M. B. Salamon, C. U. Jung, M. S. Park, K. Kim, and S. I. Lee, "Fluctuation study of the specific heat of $\mathrm{Mg}^{11} \mathrm{~B}_{2}$," Physical Review B, vol. 66, no. 13, Article ID 134515, 5 pages, 2002.

[2] J. Mosqueira, M. V. Ramallo, S. R. Currás, C. Torrón, and F. Vidal, "Fluctuation-induced diamagnetism above the superconducting transition in $\mathrm{MgB}_{2}$," Physical Review B, vol. 65, no. 17, Article ID 174522, 7 pages, 2002.

[3] A. S. Sidorenko, L. R. Tagirov, A. N. Rossolenko et al., "Fluctuation conductivity in superconducting $\mathrm{MgB}_{2}$," Journal of Experimental and Theoretical Physics Letters, vol. 76, no. 1, pp. 17-20, 2002.

[4] A. S. Sidorenko, V. I. Zdravkov, V. V. Ryazanov et al., "Twodimensional superconducting fluctuations in $\mathrm{MgB}_{2}$ films," Journal of Superconductivity, vol. 17, pp. 211-213, 2004.

[5] S. Rajput and S. Chaudhary, "Magneto-resistance investigations on the in-situ synthesized stainless steel sheathed $\mathrm{MgB}_{2}$ tapes," Journal of Superconductivity and Novel Magnetism, 2012.

[6] Q. Li and Q. Li, "Effects of vortex and critical fluctuations on magnetization of high $T_{c}$ superconductors," in Physical Properties of High Temperature Superconductors V, D. M. Ginsberg, Ed., pp. 209-264, World Scientific, Singapore, 1994.

[7] T. Ishiguro, K. Yamaji, and G. Saito, Superconductors, Springer, Berlin, Germany, 2nd edition, 1998.

[8] P. P. Freitas, C. C. Tsuei, and T. S. Plaskett, "Thermodynamic fluctuations in the superconductor $\mathrm{Y}_{1} \mathrm{Ba}_{2} \mathrm{Cu}_{3} \mathrm{O}_{9-\delta}$ : evidence for three-dimensional superconductivity," Physical Review B, vol. 36, no. 1, pp. 833-835, 1987.

[9] B. Oh, K. Char, A. D. Kent et al., "Upper critical field, fluctuation conductivity, and dimensionality of $\mathrm{YBa}_{2} \mathrm{Cu}_{3} \mathrm{O}_{7-x}$," Physical Review B, vol. 37, no. 13, pp. 7861-7864, 1988.

[10] S. J. Hagen, Z. Z. Wang, and N. P. Ong, "Anomalous in-plane paraconductivity in single-crystal $\mathrm{YBa}_{2} \mathrm{Cu}_{3} \mathrm{O}_{7}$," Physical Review $B$, vol. 38, no. 10 , pp. 7137-7140, 1988.

[11] F. Vidal, J. A. Veira, J. Maza, F. Miguélez, E. Morán, and M. A. Alario, "Probing thermodynamic fluctuations in high temperature superconductors," Solid State Communications, vol. 66 , no. 4, pp. 421-425, 1988.
[12] M. P. Fontana, C. Paracchini, C. Paris de Renzi, P. Podini, F. Licci, and F. C. Matacotta, "Temperature dependence of resistivity in $\mathrm{YBa}_{2} \mathrm{Cu}_{3} \mathrm{O}_{7-\delta}$ : study of the effect of oxygen content and of paraconductivity," Solid State Communications, vol. 69, no. 6, pp. 621-624, 1989.

[13] N. Goldenfeld, P. D. Olmsted, T. A. Friedmann, and D. M. Ginsberg, "Estimation of material parameters from the observation of paraconductivity in $\mathrm{YBaCuO}$," Solid State Communications, vol. 65 , no. 6, pp. 465-468, 1988.

[14] T. A. Friedmann, J. P. Rice, J. Giapintzakis, and D. M. Ginsberg, "In-plane paraconductivity in a single crystal of superconducting $\mathrm{YBa}_{2} \mathrm{Cu}_{3} \mathrm{O}_{7-x}$, Physical Review B, vol. 39, no. 7, pp. 42584266, 1989.

[15] R. Hopfengärtner, B. Hensel, and G. Saemann-Ischenko, "Analysis of the fluctuation-induced excess dc conductivity of epitaxial $\mathrm{YBa}_{2} \mathrm{Cu}_{3} \mathrm{O}_{7}$ films: influence of a short-wavelength cutoff in the fluctuation spectrum," Physical Review B, vol. 44, no. 2, pp. 741-749, 1991.

[16] A. V. Pogrebnyakov, L. D. Yu, T. Freltoft, and P. Vase, "Inplane paraconductivity in c-oriented epitaxial $\mathrm{YBa}_{2} \mathrm{Cu}_{3} \mathrm{O}_{7-x}$ films above $\mathrm{T}_{c}$," Physica C, vol. 183, no. 1-3, pp. 27-31, 1991.

[17] J. A. Veira and F. Vidal, "Paraconductivity of ceramic $\mathrm{YBa}_{2} \mathrm{Cu}_{3} \mathrm{O}_{7-\delta}$ in the mean-field-like region," Physica $C$, vol. 159, pp. 468-482, 1989.

[18] K. Semba, A. Matsuda, and T. Ishii, "Normal and superconductive properties of $\mathrm{Zn}$-substituted single-crystal $\mathrm{YBa}_{2}\left(\mathrm{Cu}_{1-x} \mathrm{Zn}_{x}\right)_{3} \mathrm{O}_{7-\delta}$, Physical Review B, vol. 49, no. 14, pp. 10043-10046, 1994.

[19] S. Ravi and V. Seshu Bai, "Excess conductivity studies in pure and $\mathrm{Ag}$ doped $85 \mathrm{~K}$ phase in $\mathrm{BiSrCaCuO}$ system," Solid State Communications, vol. 83, no. 2, pp. 117-121, 1992.

[20] S. Ravi and V. Seshu Bai, "Fluctuation induced excess conductivity in the $\mathrm{Bi}_{1.2} \mathrm{~Pb}_{0.3} \mathrm{Sr}_{1.5} \mathrm{Ca}_{2} \mathrm{Cu}_{3} \mathrm{O}_{y}$ compound," Physica C, vol. 182, no. 4-6, pp. 345-350, 1991.

[21] P. Mandal, A. Poddar, A. N. Das, B. Ghosh, and P. Choudhury, "Excess conductivity and thermally activated dissipation studies in $\mathrm{Bi}_{2} \mathrm{Sr}_{2} \mathrm{Ca}_{1} \mathrm{Cu}_{2} \mathrm{O}_{x}$ single crystals," Physica C, vol. 169 , no. 1-2, pp. 43-49, 1990.

[22] A. Poddar, P. Mandal, A. N. Das, B. Ghosh, and P. Choudhury, "Electrical resistivity, magnetoresistance, magnetisation, hall coefficient and excess conductivity in $\mathrm{Pb}$-doped $\mathrm{Bi}-\mathrm{Sr}-\mathrm{Ca}-\mathrm{Cu}$ oxides," Physica C, vol. 161, no. 5-6, pp. 567-573, 1989.

[23] H. M. Duan, W. Kiehl, C. Dong et al., "Anisotropic resistivity and paraconductivity of $\mathrm{Tl}_{2} \mathrm{Ba}_{2} \mathrm{CaCu}_{2} \mathrm{O}_{8}$ single crystals," Physical Review B, vol. 43, no. 16, pp. 12925-12929, 1991.

[24] D. H. Kim, A. M. Goldman, J. H. Kang, K. E. Gray, and R. T. Kampwirth, "Fluctuation conductivity of Tl-Ba-Ca-Cu-O thin films," Physical Review B, vol. 39, no. 16, pp. 12275-12278, 1989.

[25] M. O. Mun, S. Lee, M. K. Bae, and S. I. Lee, "Conductivity fluctuation in HBCCO superconductor," Solid State Communications, vol. 90, no. 9, pp. 603-606, 1994.

[26] Z. Hol’anová, J. Kačmarcik, P. Szabo et al., "Critical fluctuations in the carbon-doped magnesium diboride," Physica C, vol. 404, pp. 195-199, 2004.

[27] H. J. Kim, W. N. Kang, H. J. Kim et al., "Mixed-state magnetoresistance of c-axis-oriented $\mathrm{MgB}_{2}$ thin films," Physica C, vol. 391, no. 2, pp. 119-124, 2003.

[28] T. Masui, S. Lee, and S. Tajima, "Origin of superconductivity transition broadening in $\mathrm{MgB}_{2}$," Physica $C$, vol. 383, no. 4, pp. 299-305, 2003. 
[29] Y. Iye, "Superconducting properties associated with short coherence length-fluctuation effect and flux creep phenomenon in HTSC," in Studies of High Temperature Superconductors, A. Narlikar, Ed., vol. 1, pp. 166-181, Nova Science Publishers, New York, NY, USA, 1989.

[30] S. Rajput and S. Chaudhary, "On the superconductivity in in situ synthesized $\mathrm{MgB}_{2}$ tapes," Journal of Physics and Chemistry of Solids, vol. 69, no. 8, pp. 1945-1950, 2008.

[31] L. G. Aslamazov and A. I. Larkin, "Effect of fluctuations on the properties of a superconductor above the critical temperature," Soviet Physics, Solid State, vol. 10, pp. 875-880, 1968.

[32] L. G. Aslamasov and A. I. Larkin, "The influence of fluctuation pairing of electrons on the conductivity of normal metal," Physics Letters A, vol. 26, no. 6, pp. 238-239, 1968.

[33] W. E. Lawrence and S. Doniach, "Theory of layer structure superconductor," in Proceedings of the 12th International Conference on Low-Temperature Physics, E. Kanda, Ed., p. 361, Keigaku, Tokyo, 1971.

[34] H. J. Kim, K. H. Kim, W. N. Kang et al., "Fluctuation of superconductivity in $\mathrm{MgB}_{2}$ thin films," Physica C, vol. 408-410, no. 1-4, pp. 72-74, 2004.

[35] S. H. Han and O. Rapp, "Superconducting fluctuations in the resistivity of Bi-based 2:2:2:3," Solid State Communications, vol. 94, no. 8, pp. 661-666, 1995.

[36] U. C. Upreti and A. V. Narlikar, "Excess conductivity, critical region and anisotropy in $\mathrm{YBa}_{2} \mathrm{Cu}_{4} \mathrm{O}_{8}$," Solid State Communications, vol. 100, no. 9, pp. 615-620, 1996.

[37] M. V. Ramallo and F. Vidal, "On the width of the full-critical region for thermal fluctuations around the superconducting transition in layered superconductors," Europhysics Letters, vol. 39, no. 2, pp. 177-182, 1997.

[38] R. K. Kremer, B. J. Gibson, and K. Ahn, "Heat capacity of $\mathrm{MgB}_{2}$ : evidence for moderately strong coupling behavior," http://arxiv.org/abs/cond-mat/0102432.

[39] D. Mijatovic, $\mathrm{MgB}_{2}$ thin films and Josephson devices [Ph.D. thesis], University of Twente, Enschede, The Netherlands, 2004. 

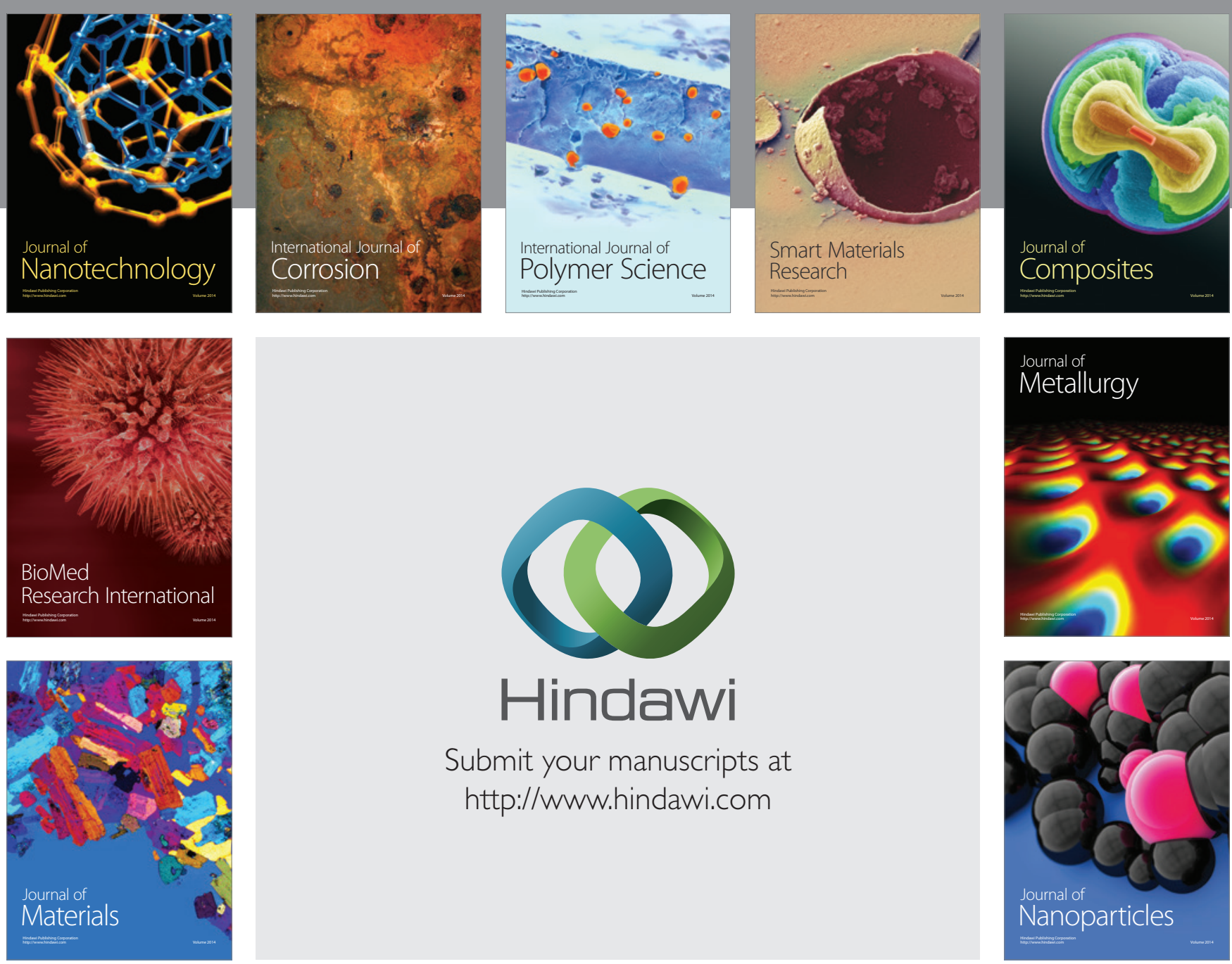

Submit your manuscripts at http://www.hindawi.com
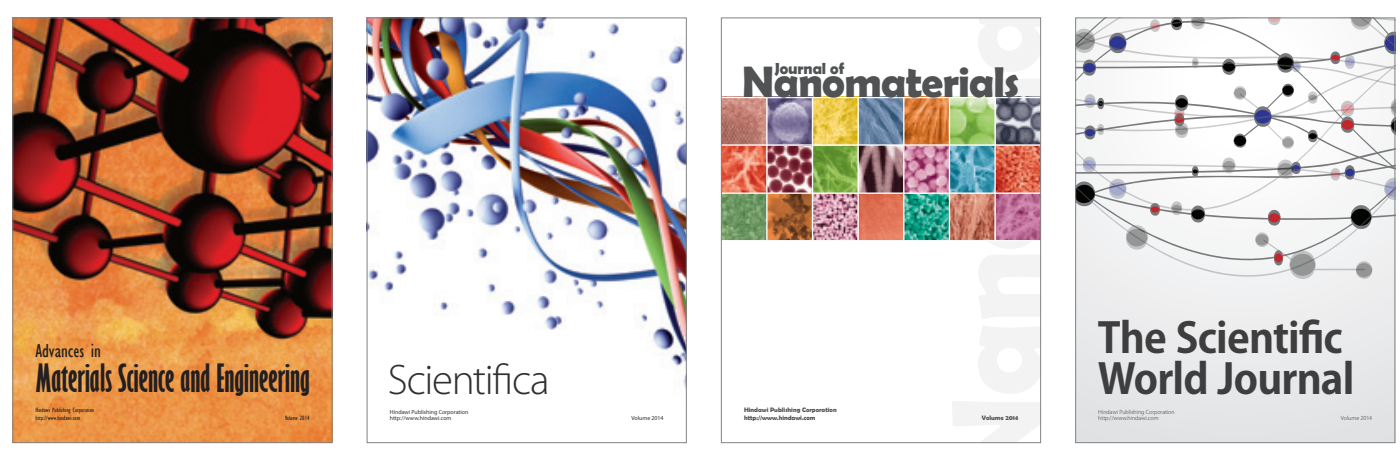

\section{The Scientific World Journal}
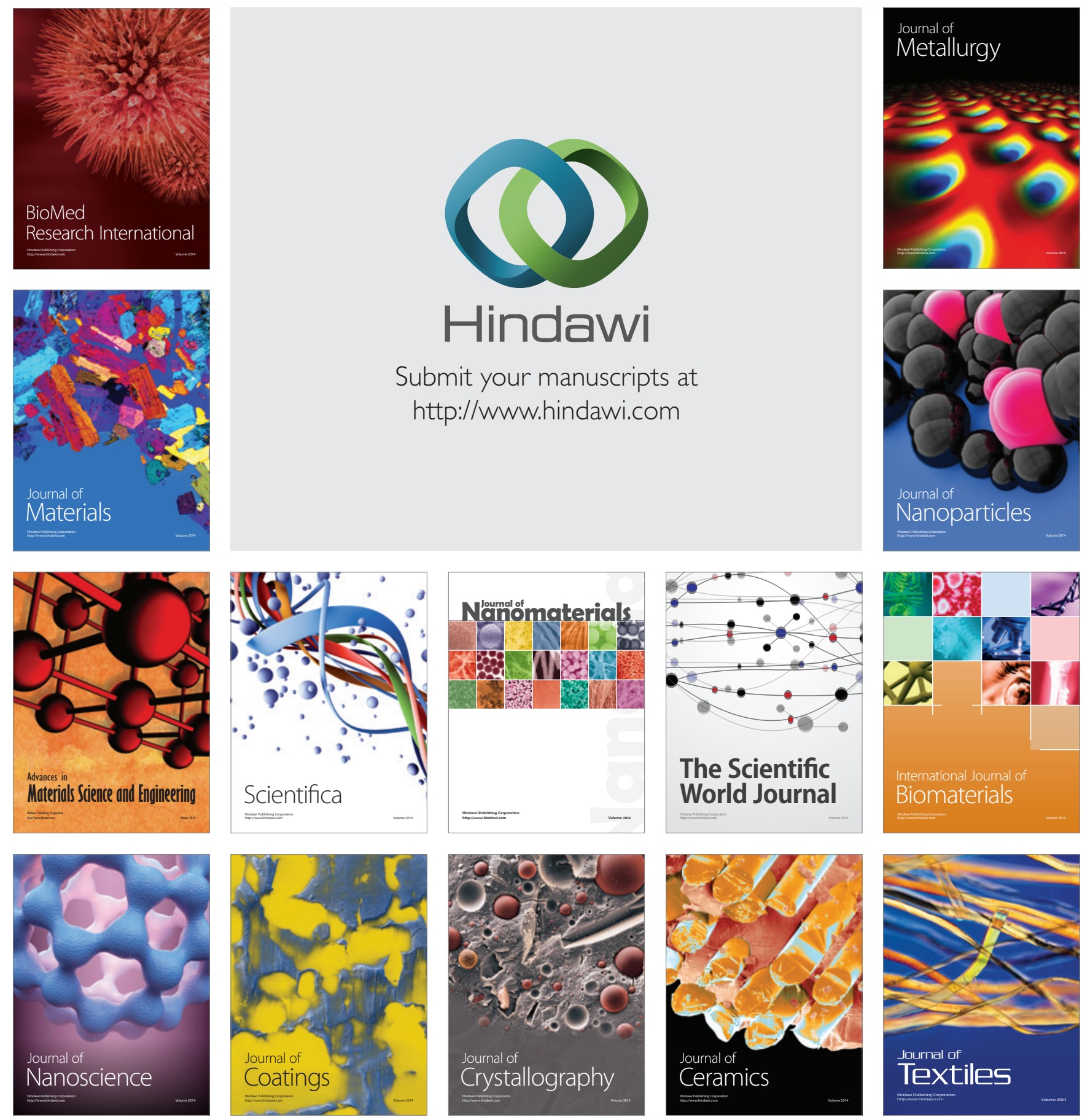